\title{
Ram Pressure Effects on the Magnetic Field of NGC 2442
}

\author{
A. Fletcher, R. Beck \\ Max Planck Institut für Radioastronomie, 53121 Bonn, Germany \\ J. Harnett \\ University of Technology Sydney, Broadway 2007, NSW, Australia \\ M. Ehle \\ XMM-Newton Science Operations Centre, ESA, 28080 Madrid, Spain \\ S. Ryder \\ Anglo-Australian Observatory, Epping, NSW 1710, Australia
}

\begin{abstract}
The $\lambda 6 \mathrm{~cm}$ radio continuum emission from the barred galaxy NGC 2442 displays several unusual features that are probably due to the interaction of the galaxy with the intergalactic medium.
\end{abstract}

New observations of the peculiar, barred galaxy NGC 2442 have been made at $\lambda 6 \mathrm{~cm}$ with the $\mathrm{ATCA}^{1}$ (Figure 1). Interesting features are revealed which probably arise from the interaction of the galaxy with the intergalactic medium.

1. The radio arms, like the optical arms,are highly deformed with the northern spiral arm bending suddenly through $\sim 90^{\circ}$.

2. Steep gradients in the radio emission at the north and western edges of the northern spiral arm indicate that the magnetic field is being compressed. Magnetic field strengths are high for spiral galaxies. The total field reaches $25 \mu \mathrm{G}$ in the northern spiral arm.

3. The regular magnetic field in the northern spiral arm is strongest on the outside - northern side - of the optical arm. The orientation of the regular field is parallel to the optical arm. The strong, offset regular field is probably due to ram pressure compressing the component of the total magnetic field that is parallel to the arm.

4. A large, isolated arm of strong linearly polarized emission $(\sim 50 \%$ polarized $)$ lies about $5 \mathrm{kpc}$ west of the nucleus. The magnetic field in this region is highly ordered and the regular field reaches about $12 \mu \mathrm{G}$.

Details and a full discussion are presented in Harnett et al. (A\&A submitted, astro-ph/0310462).

\footnotetext{
${ }^{1}$ The Australia Telescope Compact Array is part of the Australia Telescope which is funded by the Commonwealth of Australia for operation as a National Facility managed by CSIRO
} 


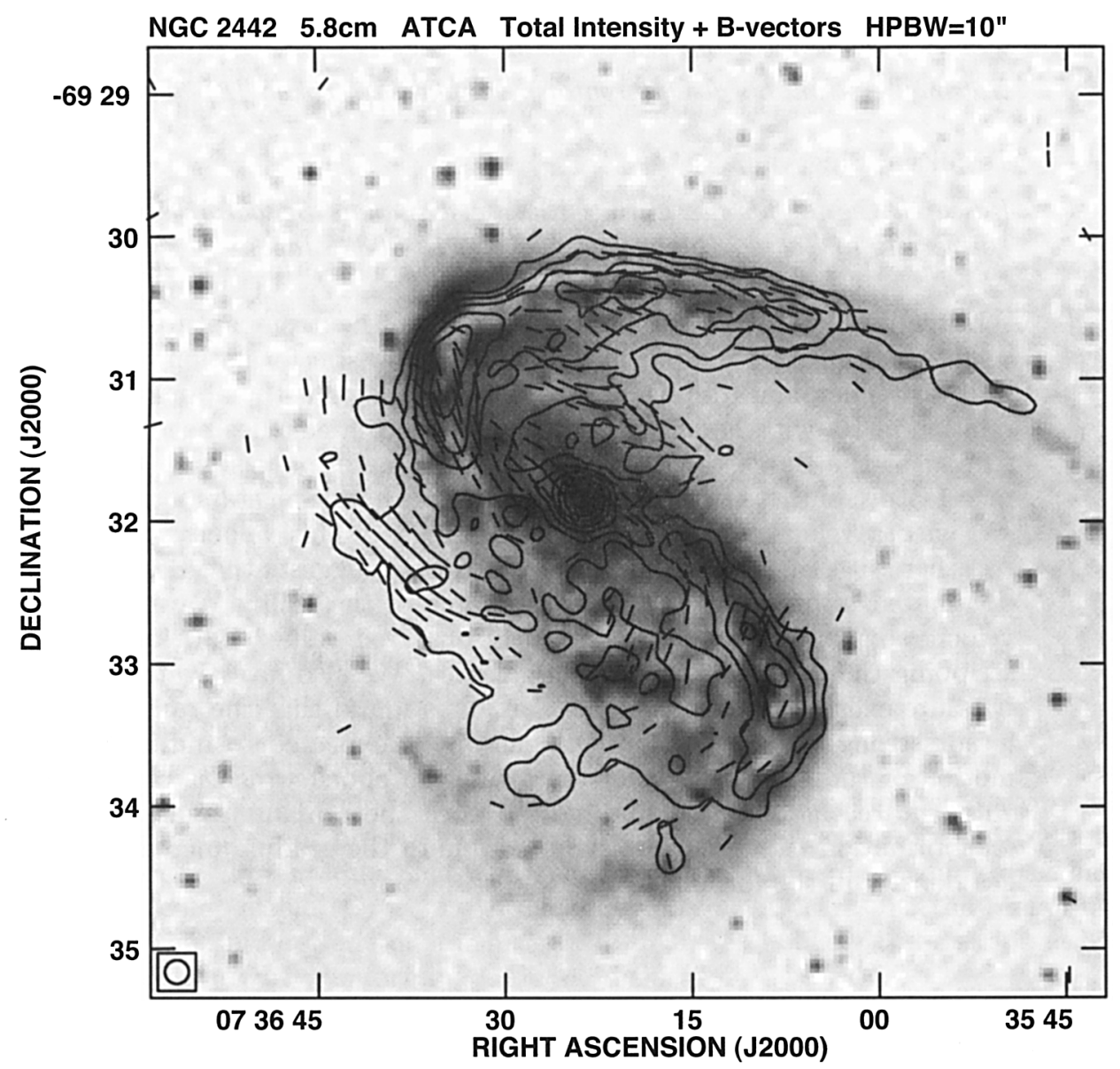

Figure 1. Total $\lambda 6 \mathrm{~cm}$ emission shown as contours overlaid on a greyscale optical image from the DSS. The half-power width of the synthesized beam is $10^{\prime \prime}$ and is shown in the bottom left corner. Contours are $1,2,4,8,16,32,64 \times$ the basic contour level of $10^{-1} \mathrm{mJy} / \mathrm{beam}$. Straight lines are E-vectors rotated by $90^{\circ}$ thus showing the magnetic field orientation, lengths scale with polarized emission and $1^{\prime \prime}$ represents $10^{-2} \mathrm{mJy} /$ beam. NGC 2442 is about $15.5 \mathrm{Mpc}$ distant and $1^{\prime \prime}$ corresponds to around $75 \mathrm{pc}$ in the galaxy. North is up. 